\title{
Isolated nail fold vasculitis in rheumatoid arthritis
}

\author{
Richard A Watts, David M Carruthers, David G I Scott
}

\begin{abstract}
Objectives-To establish the clinical significance of isolated nail fold vasculitis in patients with rheumatoid arthritis (RA). Methods-Patients attending hospital with established RA were assessed by a single observer using unaided vision. Isolated nail fold vasculitis (NFV) was diagnosed as small nail edge or nail fold lesions without any evidence of other extraarticular or systemic vasculitis. Patients were followed prospectively.

Results-Thirty patients with isolated NFV were identified and followed for a median interval of 22 months. Three patients died within three months of diagnosis and six developed complications possibly indicative of extra-articular disease: xerostomia (three), pericarditis (one), pleural effusion and empyaema (one), pulmonary fibrosis (one). No patient developed systemic vasculitis. Conclusion-Isolated NFV has a favourable prognosis compared with systemic vasculitis. There is a low risk of developing systemic or extra-articular disease.
\end{abstract}

(Ann Rheum Dis 1995; 54: 927-929)

Vasculitis is an uncommon but potentially serious complication of rheumatoid arthritis (RA), which can involve all sizes of vessel from aorta to capillaries. Isolated small nail fold or nail edge lesions (nail fold vasculitis (NFV)) reflecting mainly small vessel involvement are common and may easily be missed. These lesions are usually transient and possibly related to local trauma. ${ }^{1-3}$ As with other extraarticular manifestations of RA such as pericarditis, ${ }^{4}$ the reported incidence of these lesions reflects the intensity of the search. ${ }^{5}$ They have been reported in up to $34 \%$ of men and $18 \%$ of women with classical RA attending hospital. ${ }^{1}$ Subclinical small vessel vasculitis has been reported in up to $30 \%$ of biopsy specimens from clinically uninvolved skin taken from RA patients. ${ }^{6}{ }^{7}$ Isolated NFV is generally believed to be a benign and relatively minor feature of $\mathrm{RA},{ }^{2}$ though it may herald the onset of major arteritis, as described by Bywaters in his initial description of rheumatoid vasculitis. ${ }^{8}$ Nail fold lesions are seen in up to $40 \%$ of patients with systemic rheumatoid vasculitis (SRV). Vascular involvement, whether overt or subclinical, has been implicated in the increased mortality of RA, and therefore detection of apparently minor vasculitis may be of long term clinical significance. ${ }^{9}$

The aim of this study was to establish the clinical significance of NFV in a series of RA patients followed prospectively, and to establish their risk of subsequently developing other vasculitic or extra-articular manifestations of RA.

\section{Patients and methods}

NFV was diagnosed as the presence of small nail edge or nail fold lesions occurring in a patient with RA in whom there were no other features of SRV at the time the lesions were first observed. All patients met the American College of Rheumatology 1988 revised criteria for RA. ${ }^{10}$ Patients with a previous diagnosis of SRV were excluded. SRV was diagnosed using the Scott and Bacon criteria, namely presence of RA plus one or more of: mononeuritis multiplex or acute peripheral neuropathy; peripheral gangrene; biopsy evidence of acute necrotising arteritis plus systemic illness (fever, weight loss); and deep cutaneous ulcers or active extra-articular disease (pleurisy, pericarditis, scleritis) if associated with typical digital infarcts or biopsy evidence of vasculitis. ${ }^{11}$ Other causes of such lesions (for example diabetes mellitus and atherosclerosis) were excluded where possible. Vasculitic ulcers were diagnosed on clinical grounds-sudden onset, sharp edges and location. ${ }^{11}$ Patients with RA and vasculitis were identified from a register of all patients with vasculitis attending the rheumatology clinic at the Norfolk and Norwich Hospital. Clinical details were obtained by retrospective case note review. Patients have been seen since 1988 and assessed for the presence of NFV by a single observer (DS) using unmagnified vision in well lit conditions. They were compared with a series of patients with SRV (diagnosed as above) also seen in Norwich since 1988. Isolated NFV was not treated with disease modifying agents (DMARD) or cytotoxic drugs.

Survival curves for patients with NFV and SRV were estimated using the Kaplan-Meier product limit method. $\chi^{2}$ tests were used to compare the characteristics of patients with NFV and SRV.

\section{Results}

Since 1988, 30 patients with new onset NFV were identified and the clinical records reviewed. The table shows the demographic features of these patients and those with SRV. Both groups of patients were similar in median age (63-66 years) and median duration of RA (12-13 years) before onset of vasculitis. There were slightly more women amongst the NFV patients compared with the SRV patients, in whom there was an excess of men. Corticosteroid and DMARD use was similar in both 
Features of rheumatoid arthritis $(R A)$ patients with nail fold vasculitis (NFV) and systemic rheumatoid vasculitis (SRV)

\begin{tabular}{lll}
\hline & NFV & $S R V$ \\
\hline $\mathrm{n}$ & 30 & 47 \\
Sex (M/F) & $14 / 16$ & $25 / 22$ \\
Median duration of RA (years) & $13(1-41)$ & $12(<1-40)$ \\
Median age at diagnosis (years) & $66(34-78)$ & $63(23-81)$ \\
Median follow up (months) & $22(1-69)$ & $16(1-156)$ \\
Steroid use at diagnosis (\%) & 43 & 40 \\
DMARD use at diagnosis (\%) & 54 & 40 \\
Seropositive (\%) & 86 & 89 \\
Nodules & $14 / 20$ & $16 / 28$ \\
\hline
\end{tabular}

Values in parentheses are ranges.

groups of patients. Four patients in the NFV group and none in the SRV group were taking methotrexate when NFV was first identified.

Two patients with isolated NFV had previously documented leg ulceration of presumed venous stasis origin; one subsequently developed further ulceration at a time when there was no other evidence of vasculitis. These ulcers were not clinically of vasculitic origin and biopsy was not performed.

Six patients developed possible extraarticular complications of RA: three patients developed xerostomia with a positive Schirmer's tear test; one patient developed pericarditis two years after NFV was first observed, and a second patient developed a pleural effusion and empyaema, in both cases at a time when NFV was not present; one patient developed interstitial lung disease and died despite cyclophosphamide treatment. No other patient required cyclophosphamide.

Three patients (two women) died at three days, two months, and three months, respectively, after NFV was first noted (figure). Postmortem examination of one did not show any evidence of SRV; the second death was clinically attributed to interstitial lung disease, which had not been clinically suspected at the time NFV was first observed, and the third death was believed to be attributable to atheromatous ischaemic heart disease. The mortality rate at 24 months was $10 \%$ (95\% confidence interval $0-27 \%$ ) for the NFV patients and $27 \%$ (9-45\%) for patients with SRV. There was no significant difference in mortality between NFV and SRV patients.

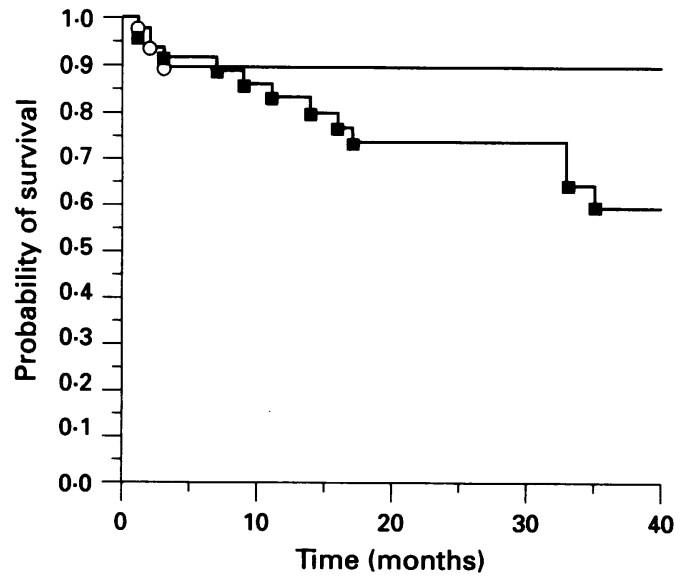

Survival probability of patients with nailfold vasculitis (O) and systemic rheumatoid vasculitis (D).

\section{Discussion}

The data suggest that isolated NFV has a favourable prognosis compared with SRV. Three patients with NFV died during the study, all within the first three months. One death was attributed to aggressive interstitial lung disease which had not been clinically apparent at the time of diagnosis of NFV and did not respond to cyclophosphamide. A postmortem examination was not performed. The other two patients died of causes probably unrelated to RA or vasculitis. The mortality of NFV appeared to be lower than that of SRV (figure), in which there was a progressive mortality. The difference in survival at 24 months was not statistically significant; this may reflect the small numbers in each group. This study did not include a group of patients with uncomplicated RA and therefore we do not know whether the prognosis for NFV is similar to or worse than that for uncomplicated RA. However, the mortality of our NFV patients was similar to previously published estimates for the survival of patients with uncomplicated RA. ${ }^{12}$

The development of isolated nail fold lesions was not considered to be an indication of DMARDs, corticosteroids, or cytotoxic drugs. The only patient to receive a cytotoxic drug was the one who developed interstitial lung disease. Corticosteroid and DMARD use at diagnosis were similar in both groups. Changes in corticosteroid dosage have been implicated in the aetiopathogenesis of vasculitis. ${ }^{13}$ Methotrexate is known to accelerate nodule formation; whether it can also precipitate vasculitis is uncertain. Because of the retrospective nature of the case note review, it was not possible to assess whether changes in corticosteroid or methotrexate dose occurred shortly before the onset of vasculitis.

Six patients with isolated NFV developed complications. Three patients developed xerostomia; labial gland biopsy specimens were not examined and it cannot be excluded that the xerostomia represented age related mucosal dryness. One patient developed pericarditis (documented as audible rub and a pericardial effusion visible on the echocardiograph). One patient developed a pleural effusion which was complicated by infection and for which surgical drainage of the empyaema was required. A further patient developed dyspnoea within four weeks of the first observation of NFV; a chest radiograph was consistent with a diagnosis of pulmonary fibrosis. A bronchoscopy and biopsy were not performed because the patient was too dyspnoeic, hence infection cannot be excluded in this patient. A clinical diagnosis of inflammatory interstitial lung disease was made and the patient received cyclophosphamide, but died subsequently. These patients did not fulfil the strict Scott and Bacon criteria for SRV, which require either the presence of NFV at the time of extra-articular disease, or histological evidence of vasculitis. ${ }^{11}$

Leg ulcers were documented in two patients before the first observation of NFV. One of these patients subsequently developed another 
leg ulcer. On clinical grounds, these were believed to be of venous stasis origin. Leg ulcers occur commonly in RA patients and are usually multifactorial. ${ }^{14}$ Vasculitic ulcers may be distinguished from the non-vasculitic form on clinical grounds. Non-vasculitic ulcers are typically painful, deeply punched out lesions of acute onset. ${ }^{11}$ They occur in sites not typically associated with venous stasis ulceration. Vasculitic ulcers are usually diagnosed by appearance and association with other evidence of vasculitis. Biopsy specimens are rarely obtained because of fears about poor wound healing. In our patients the ulcers were indolent lesions in sites typical for venous stasis ulceration.

The mechanism of NFV may differ from that of SRV. Nail fold lesions have been attributed to local trauma, ${ }^{3}$ whilst systemic vasculitis is considered to be immune mediated. ${ }^{9}$ Clinically, however, there are similarities between the two groups. Patients with NFV or SRV are similar in age and in duration of RA, and are typically seropositive for rheumatoid factor, with nodules and erosions. There is an excess of male patients in both groups, compared with the marked female preponderance in uncomplicated RA. Nail fold lesions were present in $68 \%$ of our patients with SRV. Isolated NFV and SRV may represent opposite ends of a spectrum of vasculitic manifestations in RA, with NFV representing milder 'benign' disease and SRV more severe vascular involvement. That there is little progression from benign to severe is suggested by the similarity of age and disease duration of NFV and SRV patients. There is a trend towards NFV patients being older with longer disease duration; if isolated NFV was a precursor of SRV, it would be expected that the NFV patients would be younger, with a shorter disease duration than patients presenting with SRV.

We have reported a higher annual incidence of SRV (12.5/million) than previous estimates; whether this represents a true increase, or increased diagnostic zeal is uncertain. ${ }^{15}$ This suggests, however, that RA is not becoming less severe, as has been suggested. ${ }^{16}$ The incidence of NFV is unknown and is difficult to establish because of its evanescent nature.

We conclude that development of NFV rarely heralds the imminent onset of SRV. NFV does not have the poor prognosis of SRV, and does not require aggressive immunosuppressive treatment.

1 Golding J R, Hamilton M G, Gill R S. Arteritis of rheumatoid arthritis. $B r \mathcal{F}$ Derm 1964; 77: 207-10.

2 Bywaters E G L, Scott J T. The natural history of vascular lesions in theumatoid arthritis. $f$ Chron Dis 1963; 16: 905-14.

3 Edwards J C W. Relationship between pressure and digital vasculitis in rheumatoid disease. Ann Rheum Dis 1980; 39: $138-40$.

$4 \mathrm{Kirk} \mathrm{J}$ Cosh J. The pericarditis of rheumatoid arthritis. $Q \mathcal{F}$ Med 1969; 152: 397-423.

5 Dequeker J, Rosberg G. Digital capillaritis in rheumatoid arthritis. Acta Rheum Scand 1967; 13: 299-307.

6 Wested M L, Meijer C J L, Vermmer B L, Cats A, de Vries E. Rheumatoid arthritis-the clinical significance of histo and immunopathological abnormalities in normal skin. f Rheumatol 1984; 11: 448-53.

7 Fitzgerald O M, Barnes L, Woods R, McHugh L, Barry C, O'Loughlin S. Direct immunofluorescence of normal skin in rheumatoid arthritis. Br $\mathcal{f}$ Rheumatol 1985; 24: 340-5.

8 Bywaters E G L. A variant of rheumatoid arthritis characterized by digital pad nodules and palmar fasciitis, closely resembling palindromic rheumatism. Ann Rheum Dis resembling pa.

9 Bacon P A, Kitas G D. The significance of vascular inflammation in theumatoid arthritis. Ann Rheum Dis 1994; 53: 621-3

10 Arnett F C, Edworthy S M, Block D A, et al. The American Rheumatism Association 1987 revised criteria for classification of rheumatoid arthritis. Arthritis Rheum 1988; 31: 315-23.

11 Scott D G I, Bacon P A. Intravenous cyclophosphamide plus methyl prednisolone in the treatment of systemic rheumatoid vasculitis. $A m$ f Med 1984; 76: 377-84.

12 Vollertsen R S, Conn D L, Ballard D J, Ilstrup D M, Kazmar R E, Silverfield J C. Rheumatoid vasculitis: survival and associated risk factors. Medicine 1986; 65: survival

13 Kemper J W, Baggenstross A H, Slocumbe C H. The relationship of therapy with cortisone to the incidence of vascular lesions in rheumatoid arthritis. Ann Intern Med 1957; 46: 831-5.

14 McRorie E R, Jobanputra P, Ruckley C V, Nuki G. Leg ulceration in theumatoid arthritis. Br $\mathcal{F}$ Rheumatol 1994; 33: 1078-84.

15 Watts R A, Carruthers D M, Symmons D P M, Scott D G I. The epidemiology of systemic rheumatoid vasculitis in the United Kingdom. Br f Rheumatol 1994; 33: 832-3.

16 Silman A J, Davies P, Curry H L F, Evans S J W. Is RA becoming less severe? f Chron Dis 1983; 36: 891-7. 